\title{
AORTIC REGURGITATION
}

Gerald Maurer

Heart 2006;92:994-1000. doi: 10.1136/hrt.2004.042614

Take the online multiple choice questions associated with this article (see page 1000)

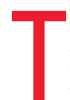
he incidence of clinically significant aortic regurgitation (AR) increases with age, typically peaking in the fourth to sixth decade of life. It is more common in men than women. The prevalence of AR in the Framingham study was reported to be $4.9 \%$, with regurgitation of moderate or greater severity occurring in $0.5 \%$.

\section{AETIOLOGY OF AORTIC REGURGITATION}

AR may be caused by malfunction of the valve leaflets themselves, by dilatation of the aortic root and annulus, or may be due to a combination of these factors (table 1). Rheumatic disease is still the most common aetiology of AR in developing countries; however, in Western Europe and North America the leading cause of AR is either congenital (particularly due to bicuspid leaflets) or degenerative disease, including annuloaortic ectasia. Understanding the mechanism leading to AR is essential for proper patient management, including the surgical approach. Thus, knowledge of the morphology of the valve leaflets, the annulus and the ascending aorta are essential.

\section{PATHOPHYSIOLOGY AND HAEMODYNAMIC CONSIDERATIONS}

AR causes volume overload of the left ventricle (LV). The total stroke volume ejected by the LV (sum of effective stroke volume plus regurgitant volume) is increased; in severe AR regurgitant volume may equal or even exceed effective stroke volume. An increase in LV end-diastolic volume is the main compensatory mechanism needed to maintain a normal effective stroke volume. Left ventricular ejection fraction is initially normal, however, LV end-diastolic pressure rises. In time LV end-diastolic volume continues to increase further and ejection fraction drops; these changes may actually precede the development of clinical symptoms. Considerable eccentric myocardial hypertrophy can occur with chronic AR and at autopsy heart weights of up to $1000 \mathrm{~g}$ have been reported.

Acute AR can be life threatening, as LV dilatation and other compensatory mechanisms cannot develop rapidly enough to avoid haemodynamic deterioration. The same regurgitant volume that would be well tolerated in chronic AR can lead to notable increases in LV end-diastolic pressure and a drop in effective stroke volume, leading to pulmonary oedema, hypotension and even cardiogenic shock

In AR there is not only volume overload but also an increase in afterload and therefore of systolic wall stress. This distinguishes AR from mitral regurgitation (MR), ${ }^{1}$ where LV volume overload is also present, but where systolic wall stress is normal or even low, since the regurgitant blood is ejected into the low pressure left atrium. Thus valve surgery in MR usually results in an increase in afterload and commonly in worsening of the LV ejection fraction, while surgical correction of AR results in a decrease in afterload and frequently an improvement of the ejection fraction. The differences in afterload between the two disorders could also explain why vasodilator treatment may be beneficial in AR, but not in MR.

\section{EVALUATION AND GRADING OF SEVERITY}

Patients with chronic AR typically remain asymptomatic for many years, with symptoms developing only in the late stages of the disease. The most common clinical complaint is shortness of breath, initially occurring during exercise, but later also at rest. Patients may be aware of a prominent, bounding heart beat and may experience sinus tachycardia at minimal exertion as well as palpitations, which can be caused by ventricular or supraventricular arrhythmias. In some instances angina can be present even in the absence of coronary artery disease.

Physical examination reveals a characteristic high-pitched, blowing decrescendo diastolic murmur and-as soon as AR becomes moderate to severe-low diastolic arterial blood pressure, widened pulse pressure, and bounding pulses. Often an increase in systolic pressure also takes place. Widened pulse pressure is a useful indicator of haemodynamically significant AR; however, its absence does not reliably exclude severe regurgitation. 
Table 1 Aetiology of aortic regurgitation (AR)

Primary valve disease
Rheumatic
$\begin{array}{ll}\text { Congenital: } & \text { Bicuspid aortic valve } \\ & \text { Outlet supracristal VSD } \\ & \text { Discrete subaortic stenosis }\end{array}$

Endocarditis*

Other inflammatory disorders

Degenerative

Traumatic leaflet rupture*

\section{Secondary AR}

Aortic root dilatation

Aortic dissection*

Damage to aortic annulus

Prolapsing intimal flap with intact leaflets and annulus

*Disorders leading to acute AR

VSD, ventricular septal defect.

\section{Diagnostic tools}

The ECG may be normal in mild AR. With greater degrees of regurgitation LV hypertrophy with or without strain pattern can be seen.

Chest $x$ ray shows evidence of LV enlargement. Dilatation of the ascending aorta and aortic knob may be seen. Aneurysmal dilatation of the aorta can be present, particularly in patients in whom the AR is related to primary disease of the aortic wall.

Echocardiography (fig 1) presently is the principal tool for diagnosis and grading of AR severity as well as for serial follow-up. Colour Doppler is a highly sensitive and specific technique for detecting $\mathrm{AR}$ and provides visualisation of the regurgitant jet. Continuous and pulsed wave Doppler offer additional haemodynamic information and aid quantitation. Importantly, two dimensional echocardiography permits evaluation of LV size and function as well as visualisation of valve structures and of the aorta. Three dimensional echocardiography may play an increasing role in obtaining more precise measurements of ventricular volumes and may offer enhanced images of valve morphology.

Aortic root angiography and cardiac magnetic resonance imaging (MRI) are alternative imaging techniques, particularly in rare instances when echocardiography is technically impossible or technically limited. Radionuclide ventriculography can be used to serially assess LV ejection fraction at rest and during exercise.

\section{Grading AR severity}

Table 2 summarises the most important parameters to be considered and has been adapted from a consensus paper recently published in the $\mathrm{US}^{2}$ and European literature.

A valuable and simple parameter for grading $\mathrm{AR}$ is measurement of the narrowest width of the proximal regurgitant jet (vena contracta) by Colour Doppler ${ }^{3}$ (fig 1). A jet width $<0.3 \mathrm{~cm}$ is highly specific for mild AR whereas a width $>0.6 \mathrm{~cm}$ is highly specific for severe AR. ${ }^{2}$ In very eccentric jets this measurement becomes unreliable.

Evaluation of the flow pattern in the proximal descending aorta using PW-Doppler yields additional important
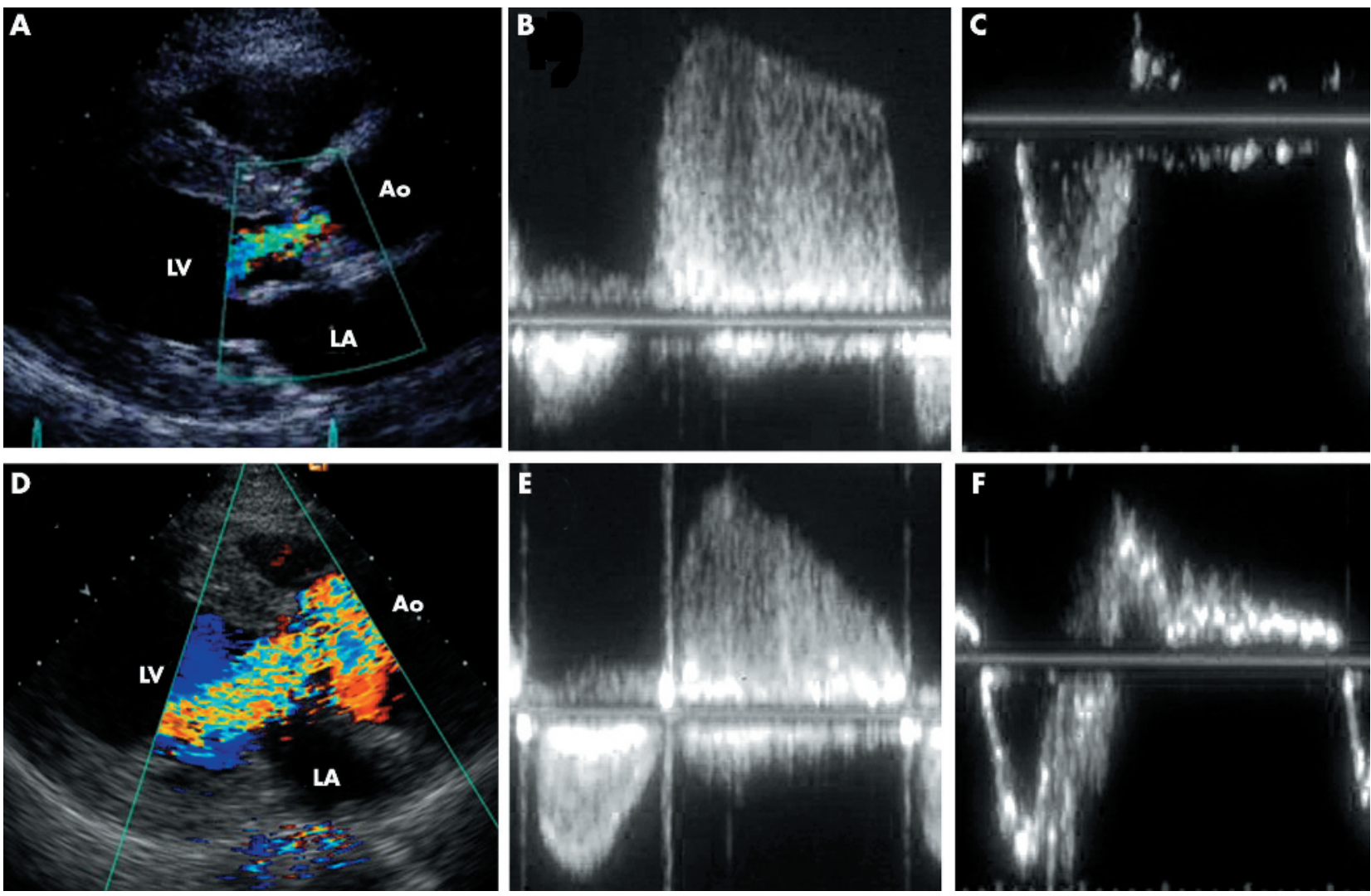

Figure 1 Echo-Doppler evaluation of aortic regurgitation (AR): panels A, B, C-mild AR; panels D, E, F-severe AR. (A) Narrow colour Doppler jet in mild AR (parasternal long axis view). (B) CW-Doppler tracing in mild AR (slow velocity decay). (C) PW-Doppler tracing from the descending aorta (suprasternal approach) in mild AR (minimal diastolic flow reversal). (D) Broad colour Doppler jet and large convergence zone in severe AR (parasternal long axis view). (E) CW-Doppler tracing in severe AR (steep diastolic velocity decay). (F) PW-Doppler tracing from the descending aorta (suprasternal approach) in severe AR (holodiastolic flow reversal). Ao, aorta; LA, left atrium; LV, left ventricle. 
Table 2 Grading of aortic regurgitation (AR)

\begin{tabular}{|c|c|c|c|c|}
\hline & Mild & \multicolumn{2}{|l|}{ Moderate } & Severe \\
\hline Specific signs for AR severity & $\begin{array}{l}\text { Vena contracta }<0.3 \mathrm{~cm}^{*} \\
\text { Central jet width }<25 \% \text { of LVOT* } \\
\text { No or brief early diastolic } \\
\text { flow reversal in descending aorta }\end{array}$ & \multicolumn{2}{|c|}{$\begin{array}{l}\text { Intermediate values } \\
\text { Intermediate values }\end{array}$} & $\begin{array}{l}\text { Vena contracta }>0.6 \mathrm{~cm}^{*} \\
\text { Central jet } \geqslant 65 \% \text { of } \mathrm{LVOT}^{*} \\
\text { Holodiastolic flow reversal in descending } \\
\text { aorta } \\
\text { Flail or wide coaptation defect }\end{array}$ \\
\hline Supportive signs & $\begin{array}{l}\mathrm{PHT}>500 \mathrm{~ms} \\
\mathrm{No} / \text { minimal flow convergence* }\end{array}$ & & & $\begin{array}{l}\text { PHT }<200 \text { ms } \\
\text { Large flow convergence* } \\
\text { Moderate or greater LV enlargement }\end{array}$ \\
\hline \multicolumn{5}{|l|}{ Quantitative parameterst } \\
\hline Reg volume (ml/beat) & $<30$ & $30-44$ & $45-59$ & $\geqslant 60$ \\
\hline Reg fraction (\%) & $<30$ & $30-39$ & $40-49$ & $\geqslant 50$ \\
\hline EROA $\left(\mathrm{cm}^{2}\right)$ & $<0.10$ & $0.10-0.19$ & $0.20-0.29$ & $\geqslant 0.30$ \\
\hline
\end{tabular}

EROA, effective regurgitant orifice area; LV, left ventricle; LVF, left ventricular function; LVOT, left ventricular outflow tract; PHT, pressure half-time; Reg, regurgitant.

*At a Nyquist limit of $50-60 \mathrm{~cm} / \mathrm{s}$.

†Quantitative parameters should be viewed with caution and only in the context of the other signs of severity because of the intrinsic limitations of quantitative measurement techniques.

information. ${ }^{2}$ Holodiastolic flow reversal is specific for severe AR (fig 1), while no or only brief diastolic flow reversal indicates mild AR.

CW-Doppler can be used to measure regurgitant flow velocity of the AR jet, which reflects the diastolic pressure gradient between the aorta and the LV. The rate of deceleration and the derived pressure half-time correspond to the rate of equalisation of these pressures. With increasing AR severity, aortic diastolic pressure decreases more rapidly, the late diastolic jet velocity becomes lower, and pressure half-time becomes shorter. A pressure half-time $>500 \mathrm{~ms}$ is usually consistent with mild AR, whereas values $<200 \mathrm{~ms}$ (according to some authors $<300 \mathrm{~ms}$ ) is considered compatible with severe $\mathrm{AR}^{2}$ (fig 1). These measurements are not always reliable, as they are affected by other causes of elevated LV diastolic pressure or low aortic diastolic pressure.

A more quantitative approach using PW Doppler is based on comparison of measurements of aortic stroke volume at the level of the LVOT with mitral or pulmonic stroke volume. ${ }^{2}$ Effective regurgitant orifice area (EROA) can be calculated from the regurgitant stroke volume and the regurgitant jet velocity time integral by CW Doppler: a regurgitant volume $\geqslant 60 \mathrm{ml}$ and EROA $\geqslant 0.30 \mathrm{~cm}^{2}$ are consistent with severe AR. Assessment of PISA (proximal isovelocity surface area) constitutes an alternative quantitative approach, although considerably less experience exists for assessment of AR than with MR. The PISA approach is limited by the interposition of valve tissue when imaging from the apex. Nevertheless, minimal or no flow convergence suggests mild regurgitation, whereas a larger flow convergence is consistent with severe AR. Quantitation using the PISA method has also been applied for $\mathrm{AR}^{4}$ and has been reported to yield regurgitant volume and, when combined with CW-Doppler measurements of jet velocity, effective regurgitant orifice area (EROA). Both quantitative methods, however, suffer from intrinsic limitations and considerable sources of error exist. Many investigators therefore recommend an integrative approach utilising all the parameters described above to provide an accurate judgement as a basis for clinical decision making.

\section{Alternative imaging tools}

Although grading is possible by Doppler echocardiography in the vast majority of patients, uncertainty may remain in some, particularly when ultrasound image quality is poor. In this case, cardiac catheterisation is still commonly used.
Invasive evaluation, however, also does not provide true quantitation, since it mostly relies on aortic root angiography which is graded semiquantitatively, as well on haemodynamic measurements. In case of uncertainty with echocardiographic grading, cardiac MRI may be a useful next step. Although regurgitant volume and regurgitant fraction can be calculated from stroke volume measurements derived from LV and RV volume estimates, the currently preferred approach involves quantification of forward and backward flow in the ascending aorta.

\section{Mechanism of AR}

Understanding the aetiology and mechanisms leading to regurgitation may be essential for proper management. Aortic valve repair, while performed infrequently at this point, may be considered in suitable cases, such as bicuspid aortic valves with leaflet prolapse.

Conversely there may be severe AR with intact aortic leaflets and a normal annulus in some cases of aortic dissection, where prolapse of the dissection membrane prevents valve closure. In such circumstances the valve may not require replacement at the time of aortic root surgery. Obtaining information about the aetiology and mechanism of AR is currently the domain of echocardiography, particularly using the transoesophageal approach. Newer imaging tools, such as three dimensional echocardiography and magnetic resonance imaging, may contribute to the assessment of the complex spatial relationships of the aortic valve structures and may ultimately improve the facility of aortic valve repair.

\section{Ascending aorta}

In all instances information about morphology and size of the ascending aorta are needed. Aortic root and annular dilatation may cause AR even when leaflets are normal. In the presence of a bicuspid aortic valve, the aortic root is frequently dilated, probably due to an abnormality of the wall, ${ }^{5}$ which may also explain the increased incidence of aortic dissection in patients with bicuspid aortic valves. Aortic root dilatation may be independent of haemodynamics and can progress further even after valve surgery. As in other types of aortic dilatation, elective surgical correction is recommended when the diameter exceeds $55 \mathrm{~mm} .{ }^{6}$

\section{Additional pathology}

Information about additional findings is also needed, including presence of abnormalities of other valves and of 
$\mathbf{A}$

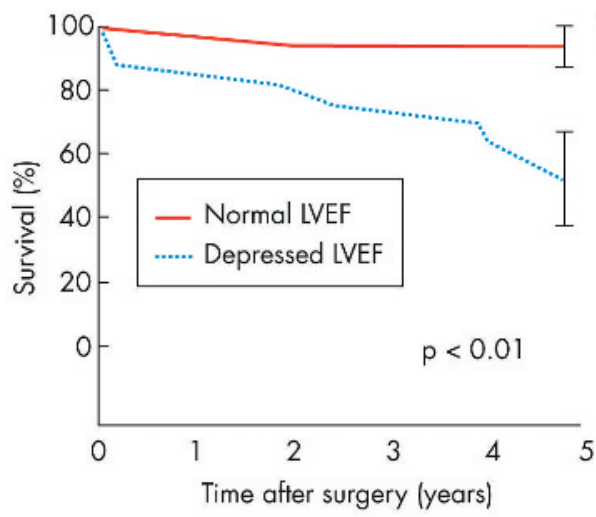

B

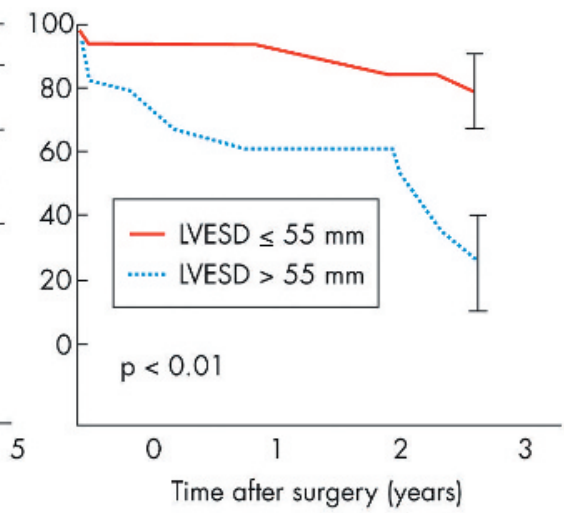

Figure 2 Postoperative survival of patients with preoperative reduced versus normal left ventricular function (panel A) and of patients with preoperative left ventricular end-systolic diameter (LVESD) $\leqslant 55 \mathrm{~mm}$ versus $>55 \mathrm{~mm}$ (panel B). LVEF, left ventricular ejection fraction. Reproduced from Bonow et al, ${ }^{810}$ with permission. endocarditis. Knowledge about possible coexistent congenital abnormalities is also essential, especially in view of the fact that some are associated with AR, such as subaortic stenosis and some forms of ventricular septal defects (VSDs).

\section{NATURAL HISTORY}

Patients with severe AR have been shown to have a significant increase in mortality and morbidity compared to the general population. ${ }^{7}$ With conservative management approximately half the surviving patients developed heart failure after 10 years and almost all others required valve surgery. Patients who became symptomatic are at increased risk; in particular, the highly symptomatic ones (New York Heart Association functional class III-IV) have been noted to have an annual mortality rate of approximately $25 \%{ }^{7}$

A diminished LV ejection fraction (below 50-55\%) is associated with reduced prognosis even in asymptomatic patients $^{78}$ (fig 2A). LV dysfunction of short duration is, however, usually reversible'; thus serial evaluation of LV function is recommended on a routine basis in these patients and surgery should be considered as soon as a drop in ejection fraction occurs. LV enlargement in and of itself also constitutes an indication for surgery ${ }^{10}{ }^{11}$ (fig 2B). The most commonly used parameters are echocardiographic endsystolic and end-diastolic diameters. ${ }^{10}$ At present ejection fraction is most commonly measured using two dimensional echocardiography or radionuclide ventriculography. For assessment of LV size actual volume measurements may increasingly replace single dimensional measurements in the future, particularly using accurate and reproducible techniques, such as MRI and three dimensional echocardiography.

\section{CLINICAL MANAGEMENT OF AORTIC REGURGITATION}

The goal of managing AR is no longer just relief of symptoms but also to provide optimal long term outcome with regard to mortality and morbidity. Achieving this goal is critically dependent on preservation of LV function and some patients may require surgical repair before onset of symptoms. In AR, criteria for early detection of myocardial damage focus on ventricular size and function. Current practice guidelines for the timing of surgery in asymptomatic patients use cut-offs derived from the published literature (table $3^{11}{ }^{12}$ ).

AR patients have an increased risk for developing endocarditis and should therefore receive antibiotic prophylaxis.

\section{Surgical options}

Valve replacement, using either a mechanical or a biological prosthesis, continues to be the mainstay of surgical treatment. The use of homografts or pulmonary autografts is

Table 3 Indication for surgery in patients with severe aortic regurgitation

\begin{tabular}{lll}
\hline Indication class* & ESC guidelinest & ACC/AHA guidelines \\
\hline I & LVEDD $>70 \mathrm{~mm}$ or & Any patient in NYHA class III or IV \\
& LVESD $>50 \mathrm{~mm}$ or & NYHA class II with normal LVF but progressive \\
& LVESI $>25 \mathrm{~mm} / \mathrm{m}^{2}$ & LF dilatation or declining EF or declining exercise tolerance on serial studies \\
& Ascending aorta $>55 \mathrm{~mm}$ & CCS class II angina \\
& NYHA class II with normal LVF and stable LVF, LV size and exercise tolerance on serial studies \\
Raprease in LV & Asymptomatic patient with normal LVF but LVESD $>55 \mathrm{~mm}$ \\
& diameters & or LVED $>75 \mathrm{~mm}$ (consider body size) \\
Bicuspid aortic valve or & EF $<25 \%$ \\
Marfan with aorta $>55 \mathrm{~mm}$ & Asymptomatic patient with normal LVF but \\
& LVESD $50-55 \mathrm{~mm}$ \\
& or LVED $70-75 \mathrm{~mm}$ (consider body size) \\
& Asymptomatic patient with decreased EF with exercise
\end{tabular}

${ }^{*}$ Class I indicates that there is evidence or general agreement that the procedure is useful; class II indicates that there is conflicting evidence or opinion; Ila indicates then that weight of evidence favours surgery whereas $\mathrm{lb}$ indicates that the efficacy of surgery is less well established. †Guidelines are only for asymptomatic patients.

ACC, American College of Cardiology; AHA, American Heart Association; CCS, Canadian Cardiovascular Society; EF, ejection fraction; ESC, European Society of Cardiology; LV, left ventricle; normal LVF, normal left ventricular function defined by EF $\geqslant 50 \%$; LVEDD, left ventricular end-diastolic diameter; LVESD, left ventricular end-systolic diameter; LVESI, left ventricular end-systolic diameter index; NYHA, New York Heart Association. 
Severe AR present

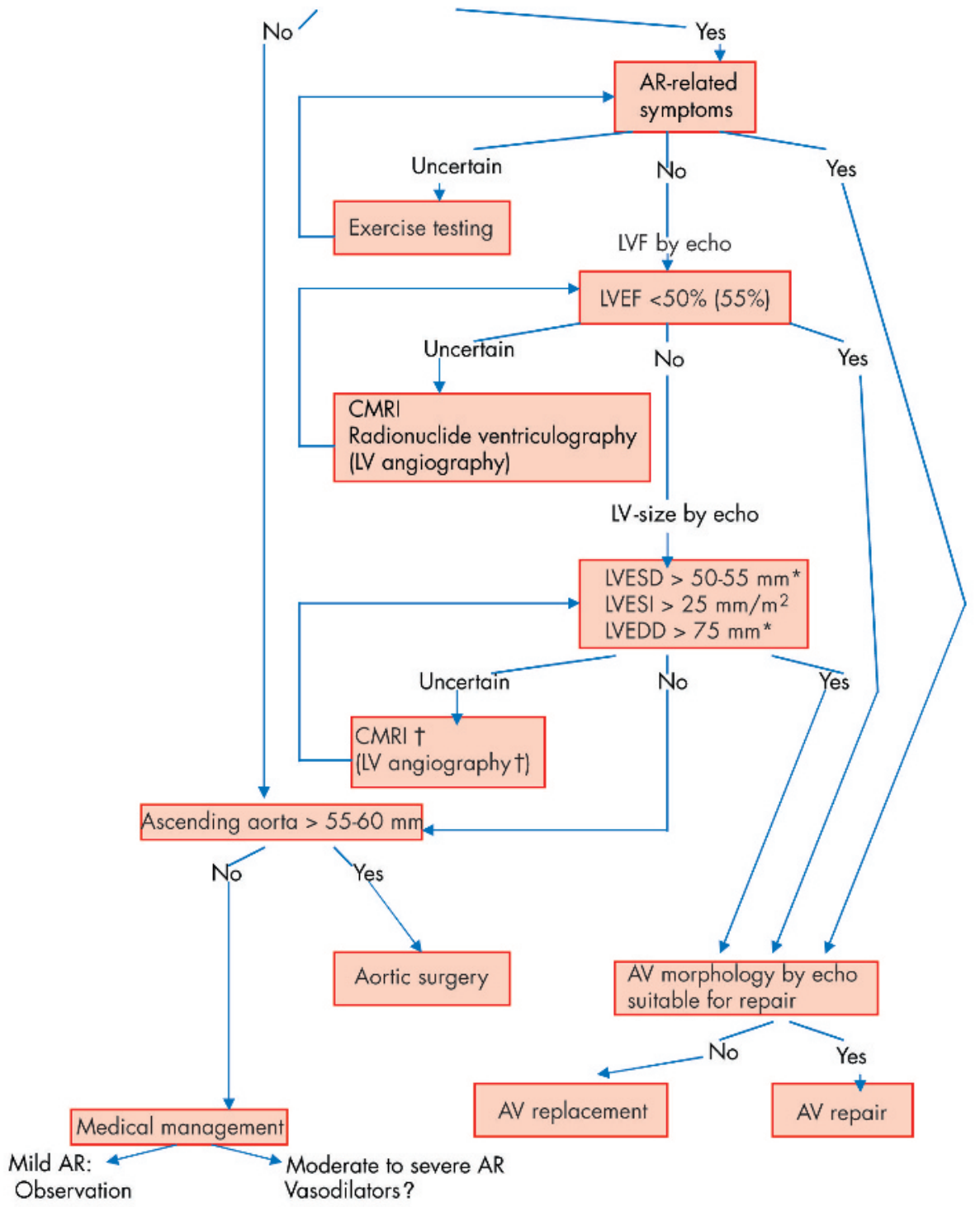

Figure 3 Management strategy for aortic regurgitation - a stepwise approach using imaging (see text). AR, aortic regurgitation; $\mathrm{AV}$, aortic valve; $\mathrm{CMRI}$, cardiac magnetic resonance imaging; $\mathrm{CT}$, computed tomography; LV, left ventricle; LVEDD, left ventricular end-diastolic diameter; LVEF, left ventricular ejection fraction; LVESD, left ventricular end-systolic diameter; LVESI, left ventricular end-systolic index. *Consider body size. †These methods use volumes rather than diameters for evaluation of left ventricle size. limited by concerns about their durability. ${ }^{13}$ Valve repair is being performed in selected patients, particularly in those with prolapsing bicuspid aortic valves and eccentric jets, ${ }^{14}$ but outcomes have generally been less favourable than for mitral valve repair.

\section{Conservative management}

Patients with mild or moderate AR can usually be managed conservatively unless surgery is indicated for correction of concomitant lesions, such as a markedly dilated aortic root. Even severe AR can be managed conservatively as long as the patient remains asymptomatic and no LV dysfunction or pronounced LV enlargement are present. Patients with moderate to severe AR often receive vasodilator treatment, particularly when the ventricle is already dilated. One study comparing nifedipine with digoxin ${ }^{15}$ found the patients on nifedipine developed fewer symptoms and less LV dysfunction; however, no placebo group was included and the number of included patients was small. Similar benefits have been reported with angiotensin converting enzyme inhibitors and other vasodilators, although these trials also included only small numbers of patients. In a recent randomised trial ${ }^{16}$ in 95 patients with asymptomatic severe AR comparing nifedipine or enalapril to placebo, vasodilators did not reduce or delay the need for aortic valve replacement and did not reduce regurgitant volume, did not decrease LV size, and did not improve LV function. Thus, in spite of the fact that some trials suggest a benefit from using vasodilators in AR, evidence supporting their use must be considered limited. ${ }^{17}$

\section{A step by step approach to managing the patient with aortic regurgitation}

After aortic regurgitation has been detected by physical examination or by echocardiography the next step is to assess its severity (fig 3). This is most commonly done by echoDoppler; in case of uncertainty, cardiac MRI (CMRI) should preferably be used. Aortography can be an alternative. Even in the absence of severe AR, prophylactic surgery may be 


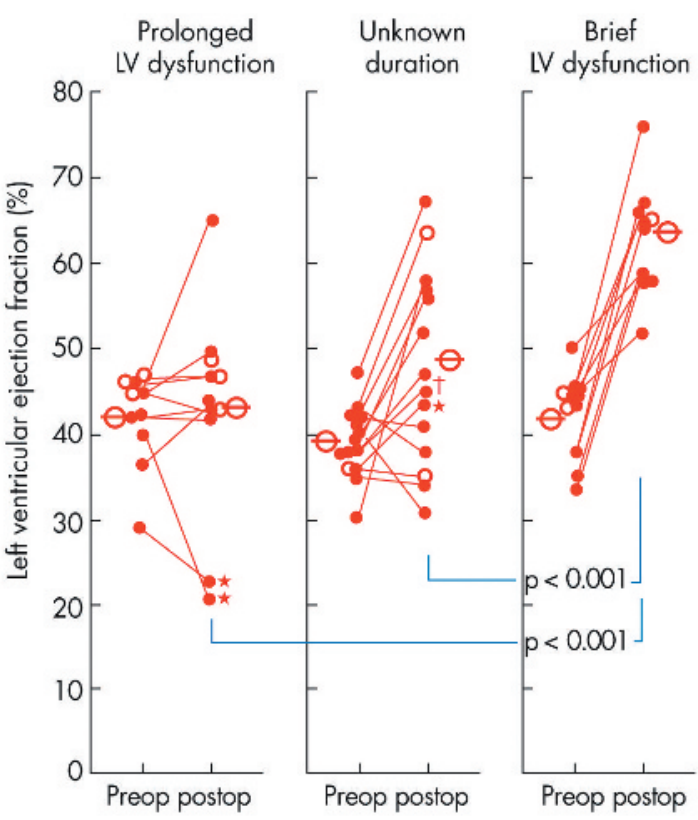

Figure 4 Change in left ventricular (LV) ejection fraction at rest preand postoperatively in patients with severe aortic regurgitation and prolonged LV dysfunction (>14 months), LV dysfunction of unknown duration, and brief LV dysfunction ( $\leqslant 14$ months). Reproduced from Bonow et al, ${ }^{9}$ with permission.

needed for aortic root aneurysm, and this requires further evaluation of the ascending aorta. Surgery is recommended when the maximum diameter reaches 55-60 mm. If echocardiography cannot provide reliable measurements, CMRI or computed tomography (CT) should be performed.

In the case of severe AR, the next question is about symptoms. In their presence, surgery is indicated. ${ }^{18}$ Exercise testing may be helpful to clarify symptom status.

In a definitely asymptomatic patient, the next step is to assess LV function. In the case of reduced LV function as defined by ejection fraction $<50 \%$ (some even recommend $<55 \%$ ), surgery should be performed. ${ }^{19}$

Asymptomatic patients with normal LV function should be considered for valve surgery for preservation of myocardial function in presence of pronounced LV enlargement, ${ }^{20}$ particularly when the end-systolic diameter exceeds $55 \mathrm{~mm}^{11}$ ( $50 \mathrm{~mm}$ by European guidelines ${ }^{12}$ ) or when the end-diastolic diameter of the LV exceeds $75 \mathrm{~mm}$. These cutoff values must take body size into account. They are valid for average-sized men but may be too large for women, especially if the patient is small. For this reason, some prefer a cut-off $\mathrm{LV}$ end-systolic index $>25 \mathrm{~mm} / \mathrm{m}^{2}$. However, indexing has its own limitations and both the absolute value and index must always be viewed on the background of individual patient size. Uncertain echo measurements may require clarification by CMRI or LV angiography.

If surgery is indicated for any of the above mentioned reasons, echocardiography should evaluate whether a valve repair is feasible or whether valve replacement must be performed.

In addition, aortic size and morphology must be assessed in order to evaluate the need for concomitant aortic surgery.

If surgery is not indicated, serial clinical and echocardiographic evaluation is required in patients who remain asymptomatic. In stable patients, one year follow-up intervals may be appropriate as studies have shown that LV dysfunction developing over 12-14 months is usually reversible ${ }^{9}$ (fig 4).

In compliance with EBAC/EACCME guidelines, all authors participating in Education in Heart have disclosed potential conflicts of interest that might cause a bias in the article.

\section{REFERENCES}

1 Wisenbaugh T, Spann JF, Carabello BA. Differences in myocardia performance and load between patients with similar amounts of chronic aortic versus chronic mitral regurgitation. J Am Coll Cardiol 1984;3:916-23.

2 Zoghbi WA, Enriquez-Sarano M, Foster E, et al. Recommendations for evaluation of the severity of native valvular regurgitation with two-dimensional and Doppler echocardiography. J Am Soc 2003;16:777-802.

- Guideline recommending the use of the various echo-Doppler parameters for grading AR severity.

3 Tribouilloy CM, Enriquez-Sarano M, Bailey KR, et al. Assessment of severity of aortic regurgitation using the width of the vena contracta: a clinical color Doppler imaging study. Circulation 2000;102:558-64.

4 Tribouilloy CM, Enriquez-Sarano M, Fett SL, et al. Application of the proximal flow convergence method to calculate the effective regurgitant orifice area in aortic regurgitation. J Am Coll Cardiol 1998;32:1032-9.

5 Bonderman D, Gharehbaghi-Schnell E, Wollenek G, et al. Mechanisms underlying aortic dilatation in congenital aortic valve malformation. Circulation 1999;99:2138-43.

6 Davies RR, Goldstein U, Coady MA, et al. Yearly rupture or dissection rates for thoracic aortic aneurysms: simple prediction based on size. Ann Thorac Surg 2002;73:17-28

7 Dujardin KS, Enriquez-Sarano M, Schaff HV, et al. Mortality and morbidity of aortic regurgitation in clinical practice: a long-term follow-up study. Circulation 1999:99:1851-7.

- Study of long term outcome of $\mathbf{2 4 6}$ patients with severe or moderately severe $A R$, showing excess mortality and high morbidity.

8 Bonow RO, Picone AL, Mclntosh CL, et al. Survival and functional results after valve replacement for aortic regurgitation from 1976 to 1983: impact of preoperative left ventricular function. Circulation 1985;72:1244-56.

9 Bonow RO, Rosing DR, Maron BJ, et al. Reversal of left ventricular dysfunction after aortic valve replacement for chronic aortic regurgitation: influence of duration of preoperative left ventricular dysfunction. Circulation 1984;70:570-9.

- Demonstrates that duration of preoperative LV dysfunction is an important determinant of reversibility of LV dysfunction after aortic valve replacement.

10 Bonow RO, Rosing DR, Kent KM, et al. Timing of operation for chronic aortic regurgitation. Am J Cardiol 1982;50:325-36.

11 Bonow R, Carabello B, DeLeon AC Jr, et al. Guidelines for the management of patients with valvular heart disease: executive summary: a report of the American College of Cardiology/American Heart Association task force on practice guidelines (committee on management of patients with valvular heart disease). Circulation 1998;98:1949-84.

- American College of Cardiology/American Heart Association guidelines for management of patients with valvular heart disease.

12 lung B, Gohlke-Barwolf $C$, Tornos $P$, et al. Recommendations on the management of the asymptomatic patient with valvular heart disease. Eur Heart J 2002;23:1253-66

- European guidelines for managing patients with asymptomatic valvular heart disease.

13 Kouchoukos NT. Aortic allografts and pulmonary autografts for replacement of the aortic valve and the aortic root. Ann Thorac Surg 1999;67:1846-8.

14 Nash PJ, Vitvitsky E, Li J, et al. Feasibility of valve repair for regurgitant bicuspid aortic valves-an echocardiographic study. Ann Thorac Surg 2005;79:1473-9.

- Successful repair of regurgitant bicuspid aortic valves described to be more feasible for those patients with eccentric regurgitant jets, and in absence of cusp or commissural thickening or cusp calcification.

15 Scognamiglio R, Rahimtoola SH, Fasoli G, et al. Nifedipine in asymptomatic patients with severe aortic regurgitation and normal left ventricular function. N Engl J Med 1994;331:689-94.

16 Evangelista A, Tornos P, Sambola A, et al. Long-term vasodilator therapy in patients with severe aortic regurgitation. N Engl J Med 2005;353:1342-9.

- Randomised trial in 95 patients with asymptomatic severe AR comparing nifedipine or enalapril to placebo. Vasodilators did not reduce or delay the need for aortic valve replacement, nor did they reduce regurgitant volume, decrease LV size or improve LV function.

17 Carabello BA. Vasodilators in aortic regurgitation - what is the evidence of their effectiveness? N Engl J Med 2005;353:1400-2.

18 Klodas E, Enriquez-Sarano M, Tajik AJ, et al. Optimizing timing of surgical correction in patients with severe aortic regurgitation: role of symptoms. J Am Col Cardiol 1997;30:746-52.

- In the setting of severe AR, preoperative functional class III or IV symptoms are independent risk factors for excess immediate and long term postoperative mortality. Class II symptoms should be a strong incentive to consider immediate surgical correction of severe AR. 
19 Chaliki HP, Mohty D, Avierinos JF, et al Outcomes after aortic valve replacement in patients with severe aortic regurgitation and markedly reduced left ventricular function. Circulation 2002;106:2687-93.

- Study showed excess perioperative mortality in patients with severe AR and low ejection fraction. In spite of this in most patients there was improvement in postoperative ejection fraction and long postoperative survival without recurrence of heart failure.
20 Klodas $\mathbf{E}$, Enriquez-Sarano M, Taijk AJ, et al. Aortic regurgitation complicated by extreme left ventricular dilatation: longterm outcome after surgical correction. J Am Coll Cardiol 1996;27:670-7.

- Extreme LV dilation due to AR is frequently associated preoperatively with a reduced ejection fraction but is not a marker of irreversible LV dysfunction. Extreme LV dilation is not a contraindication to surgery, which should be performed before left ventricular dysfunction occurs.

\section{MULTIPLE CHOICE QUESTIONS}

Education in Heart Interactive (www.heartinl.com/misc/education.shtml)

There are six multiple choice questions associated with each Education in Heart article (these questions have been written by the authors of the articles). Each article is submitted to EBAC (European Board for Accreditation in Cardiology; www.ebac-cme.org) for 1 hour of external CPD credit.

How to find the MCQs: Click on the Online Learning: [Take interactive course] link on the table of contents for the issue online or on the Education in Heart collection (www.heartinl.com/cgi/collection/heart_education).

Free access: This link will take you to the BMJ Publishing Group's online learning website. Your Heart Online user name and password will be recognised by this website. As a Heart subscriber you have free access to these MCQs but you must register on the site so you can track your learning activity and receive credit for completed courses.

How to get access: If you have not yet activated your Heart Online access, please do so by visiting http://www.bmijournals.com/cgi/activate/basic and entering your six digit (all numeric) customer number (found above your address label with your print copy). If you have any trouble activating or using the site please contact subscriptions@bmigroup.com

Case based Heart: You might also be interested in the interactive cases published in association with Heart (http://cpd.bmijournals.com/cgi/hierarchy/cpd_node;CBH) 